\title{
COMMUNICATION STANDARDS FOR DISTRIBUTED RENEWABLE ENERGY SOURCES INTEGRATION IN FUTURE ELECTRICITY DISTRIBUTION NETWORKS
}

\author{
Anish Jindal, Angelos K. Marnerides, Antonios Gouglidis, Andreas Mauthe, David Hutchison \\ InfoLab21, School of Computing \& Communications, Lancaster University, Lancaster, UK \\ E-mail: a.jindal3@lancaster.ac.uk, angelos.marnerides@lancaster.ac.uk, \\ a.gouglidis@lancaster.ac.uk, a.mauthe@lancaster.ac.uk, d.hutchison@lancaster.ac.uk
}

\begin{abstract}
Distributed Renewable Energy Sources (DRESs) such as wind and solar are becoming a promising alternative for the energy supply in modern (smart) electricity grids as part of future sustainable smart cities. Successful integration of DRESs requires efficient, resilient, and secure communication in order to satisfy the highly challenging and real-time constraints of smart city applications. Regardless of the various research solutions proposed in this context within the last decade, the relevant standardization is a non-trivial issue and is still in its infancy. In this position paper, we briefly review the currently employed DRES communications standards and identify the gaps in their present status. Finally, we discuss and suggest potential pathways for further improvement.
\end{abstract}

Index Terms - Communication standards, communication signals, distributed renewable energy sources, smart city.

\section{INTRODUCTION}

Conventional electricity distribution networks are experiencing a rapid transition towards modern (smart) grids as they begin to rely heavily on the efficiency of the underlying communication infrastructure. The smart grid ecosystem aims to support highly demanding real-time applications (e.g. electric vehicles and balancing demand \& supply in smart cities) and its generic architecture considers Distributed Renewable Energy Sources (DRESs) to act as an integral element. Hence, an increased trend towards the integration of DRESs in currently deployed energy networks is undertaken in developed and developing countries. Nonetheless, the integration of DRESs promotes a highly distributed environment that eventually triggers a range of challenges in the context of real-time communication between the various smart grid operational layers and smart city applications. Thus, the DRES communications are required to fulfil or assist a variety of requirements including notably resilience, security, stability, and interoperability.

As depicted in Fig. 1, the smart grid is decomposed into various entities pertaining to control \& monitoring, energy generation, transmission \& distribution, and end-users. The

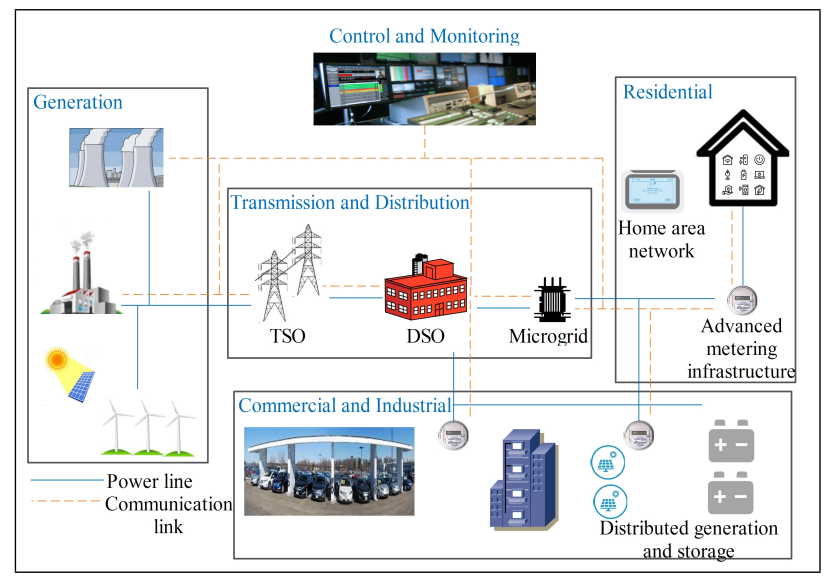

Fig. 1. Overall communication architecture of the smart grid.

control and monitoring unit is where the collected data is analyzed to take decisions, the energy generation can be from conventional sources and/or DRESs. The transmission system operator (TSO) and distribution system operator (DSO) are mainly responsible to provide energy to the end-users (such as residential and commercial sectors) directly or via the microgrid. The end-user sector can also have DRESs providing local support. All these units are connected to each other through protocols running over the power and communication link [1]. Hence, a critical requirement in smart grid relates to the timely delivery of data to the utility operator and various communication protocols/standards are available specified for the energy sector. Such protocols/standards explicitly specify how the devices should communicate their data to the local controller or the service provider.

The diversity of communication standards at the various layers of interaction (e.g., TSO to DSO level and DSO to microgrid level) due to the openness of the energy service and manufacturing market makes data collection, storage and processing a quite challenging domain. Consequently, tasks related to real-time decision making for a range of smart city applications (e.g., end-user billing, demand-response) as well as critical domains such as infrastructure security and resilience are affected. We argue, that even by restricting the view in 
currently employed communication standards that aim to integrate DRESs, several issues exist that are required to be addressed in the context of composing a unified, cross-platform, and end-to-end communication standard. Thus, this paper reviews various available international standards in the market for the purpose of data transmission at the local DRES controller or the service provider. The paper highlights the current gaps within existing standards and further provides crucial viewpoints for future development.

\section{RELEVANT STANDARDS}

A range of organizations and governmental bodies produced a variety of communication standards in the energy sector that are currently adopted by manufacturers and vendors.

\subsection{IEEE Standards}

\subsubsection{IEEE $1547-2018$}

The IEEE 1547-2018 [2] describes the "Standard for Interconnection and Interoperability of Distributed Energy Resources with Associated Electric Power Systems Interfaces". The purpose of this standard is to define the connection interface between the local DRESs and local area electric power systems at primary and distributed levels of voltage. For example, it specifies how the smart inverter injects reactive power to the system voltage when the latter decreases. In addition to it, this standard specifies the response to local area power systems in abnormal conditions. To enable communication capabilities, the standard states that the local DRES shall have provisions for communication and should conform to existing standards such as IEEE 2030.5, IEEE 1815 or SunSpec Modbus. However, it does not specify the explicit communication requirements for DRESs to communicate with the grid at TSO/DSO level and their impact on the overall security and optimal performance of the grid.

\subsubsection{IEEE 1459-2010}

IEEE 1459-2010 outlines "Definitions for the Measurement of Electric Power Quantities Under Sinusoidal, Nonsinusoidal, Balanced, or Unbalanced Conditions" [3]. The proposed standard is an improved version of the original IEEE 1459 standard produced in 2000. It provides new definitions related to various scenarios in an organized manner, which can be used while designing the new devices for the purpose of energy quantification. These scenarios cover a wide range of applications varying from situations where voltage and current waveforms do not conform to the sinusoidal oscillation, unbalanced load, asymmetrical supplying voltages, etc. All these definitions apply to single-phase sinusoidal and non-sinusoidal, three-phase (balanced and unbalanced current and voltage waveforms) sinusoidal and nonsinusoidal and can be used to test the effectiveness of the various designed and developed algorithms and instruments. However, this standard cannot be used for the purpose of designing real-time control of dynamic devices which detect anomalies in the system.

\subsubsection{IEEE 1564-2014}

The "IEEE Guide for Voltage Sag Indices" [4] presents various indices and characteristics for evaluating the voltage sags in the electrical power systems caused due to faults, transformer or feeder energizing. A voltage sag can be described as a very short term voltage variation triggered by voltage reduction disrupting the normal working operation of the enduse devices. This standard helps in understanding the severity of the voltage sag by quantifying the individual root-mean square voltage variation events occurring at a particular location or the whole power system (using the single-site and system indices). The standard is not directly targeting the integration of the DRESs in smart grids on its own. However, it can help in evaluating the voltage sags caused by the their integration. Nevertheless, the standard does not comment on real-time reporting and assessment of power system and end-user devices suffering from voltage sags, which can potentially cause security and resilience issues.

\subsection{International Electrotechnical Commission (IEC) Standards}

\subsubsection{IEC 61400-25-2:2015}

The "Communications for monitoring and control of wind power plants Information models" standard was published in 2015 [5]. It defines information gathering and exchange models with their communication functionality to reach interoperability in the wind power plants components such as wind turbines. Since data is generated by intelligent devices in the wind power plants, a compatible device level model is required for providing domain-specific services. In this regard, it gives the definitions of the logical classes (e.g., device class, node class, and data class) associated with syntax and semantics that should be considered to make these models independent of the particular protocol suites, implementation and the operating systems. However, it does not specifically describe the mapping of these classes to the explicit communication standards required for real-time information sharing.

\subsubsection{IEC 62052-11:2003 (Amended 2016)}

The IEC 62052-11 standard deals with "Electricity metering equipment $(\mathrm{AC})$ General requirements, tests and test conditions", specifically focused to the metering equipment [6]. It was originally proposed in 2003 and later amended in 2016. As metering equipment would be present in every entity of the smart grid to gather the value of power generated or used, 
this standard provides benchmark definitions related to their functional and mechanical elements. Moreover, it defines various aspects related to insulation, quantities associated with the meter as well as for the tests. It also highlights the differences between meters to be used in indoors and outdoors. The standard mostly covers tests related to indoor/outdoor metering equipment applied to recently installed infrastructure for measuring energy in 50 and $60 \mathrm{~Hz}$ networks. However, it does not specify the requirements for data interfaces to the register of meters for the end-to-end delivery of data.

\subsubsection{IEC 62053}

The IEC 62053 standard has various parts of which each deals with the different aspects of "electricity metering equipment (AC)". For instance, part 11 deals with the "Electromechanical meters for active energy (classes 0,5, 1 and 2)", part 21 deals with the requirements for "Static meters for active energy (classes 1 and 2)", part 22 discusses about the "Static meters for active energy (classes $0,2 \mathrm{~S}$ and 0,5 S)", part 23 presents guidelines for "Static meters for reactive energy", and part 24 highlights the aspects related to "Static meters for reactive energy at fundamental frequency (classes $0,5 \mathrm{~S}, 1$ $\mathrm{S}$ and 1)". The standard presents technical guidelines for the static meters for various purposes, however, the present market scenario is more focused towards the use of smart meters. Therefore, the standard needs to be updated with respect to providing the measurements of the specified energies in different parts for smart meters.

\subsection{European Union Standards}

\subsubsection{Regulation $2016 / 631$}

The European Union specified a regulation in 2016 which focuses on "establishing a network code on requirements for grid connection of generators" [7]. Although it is not a standard, the 2016/631 regulation lists important definitions and provides specifications on the establishment of network elements towards the grid connection of generators (such as synchronous power-generating modules and power park modules) which is pivotal in the smart grid. The proposed EU policy emphasizes four types of power generation modules namely type $\mathrm{A}$, type $\mathrm{B}$, type $\mathrm{C}$, and type $\mathrm{D}$ with each module having different threshold limits of maximum capacity depending on their area of operation. It also specifies the regulation aspects when multiple TSOs are present. Apart from this, the regulation also provides the general requirements for such modules, for example, the time duration for which the module can operate on variable frequency with deviation from the normal value without the need to disconnect it from the electric network. This regulation has to be considered when generators are to be connected to a given grid network.

\subsubsection{CLC/TS 50549}

The CLC/TS 50549 technical standard was proposed in 2015 and specifies the "Requirements for generating plants to be connected in parallel with distribution networks" and is divided into two parts. These plants can use DRESs for power generation and using this standard, they can connect to the low or medium voltage distribution networks. The first part of the suggested standard describes the "Connection to a LV distribution network above $16 \mathrm{~A}$ " and deals with scenarios involving the conversion of an energy source into $\mathrm{AC}$, the connection of low-voltage distribution network above $16 \mathrm{~A}$ and their paralleled operation. Moreover, the first part also specifies the requirement for power plants in terms of their connection scheme, choice of switchgear, nominal operating range (of frequency and voltage), effect of disturbances on the network, and response to variations. The second part of the standard emphasizes on the "Connection to a MV distribution network" and describes the scenarios dealing with the medium voltage distribution network instead of low-voltage. Moreover, part 2 also outlines the type of data sent for the remote monitoring and information exchange between the power plant and the control server with respect to data, type of signal, purpose, and refresh time. However, it does not include impact and connection assessment of the power system, and the islanded operation of the power plants.

\subsubsection{EVS 50438:2013}

The EVS 50438:2013 standard provides the "Requirements for micro-generating plants to be connected in parallel with public low-voltage distribution networks" [8]. It specifies the technical details of the functionality and operational capabilities of power plants that are run in parallel with low-voltage distribution networks. It also provides the national level prescribed specifications of operational voltage, frequency, and protection settings which are to be followed in different European countries for connecting power plants to the low-voltage networks. This standard can be used by the service providers while integrating DRESs in the low-voltage networks at the micro-grid level. However, the requirements for the security, resilience, and interoperability needs to be described before actually integrating DRESs in the network.

\section{PRESENT STATUS, GAPS AND SUGGESTIONS}

As discussed in the previous section, there exist many international standards in the electricity market laying foundations to different aspects of the DRESs integration in the electricity network. However, there are still aspects that have not been sufficiently considered or are completely absent. Table 1 summarizes the present status, gaps, and provide suggestions to improve the integration of DRESs based on the standards discussed earlier. 
Table 1. Present status, gaps, and suggestions for further improvement.

\begin{tabular}{|c|c|c|c|}
\hline Standard & Description & Gaps & Suggestions \\
\hline IEEE 1547-2018 & $\begin{array}{l}\text { Interconnection specifications for a } \\
\text { DRES to connect to local power sys- } \\
\text { tem interfaces }\end{array}$ & $\begin{array}{l}\text { Use of DRESs at the end-user level simultane- } \\
\text { ously with TSO/DSO level was not considered. }\end{array}$ & $\begin{array}{l}\text { 1) Existing standard needs to consider the different in- } \\
\text { tegration requirements at TSO/DSO level. } \\
\text { 2) Comment on mitigation of cyber-security issues on } \\
\text { DRES interfaces by specifying specific communica- } \\
\text { tion guidelines. }\end{array}$ \\
\hline IEEE $1459-2010$ & $\begin{array}{l}\text { Measurement of Electric Power } \\
\text { Quantities }\end{array}$ & $\begin{array}{l}\text { Cannot be used for designing real-time control } \\
\text { of dynamic devices which detect anomalies in } \\
\text { the system as no generalized power theory was } \\
\text { available when it was created. }\end{array}$ & $\begin{array}{l}\text { Can be used when new DRES infrastructures need to } \\
\text { be installed to measure its performance, if the gaps are } \\
\text { addressed. }\end{array}$ \\
\hline IEEE 1564-2014 & Voltage Sag Indices & $\begin{array}{l}\text { Reporting and assessment of voltage sags is not } \\
\text { clearly described. }\end{array}$ & $\begin{array}{l}\text { Specify requirements for transmission of losses due to } \\
\text { voltage sags for strengthening security and resilience. }\end{array}$ \\
\hline $\begin{array}{l}\text { IEC } \quad 61400-25-2: \\
2015\end{array}$ & $\begin{array}{l}\text { Information models of communica- } \\
\text { tion for monitoring and control of } \\
\text { wind power plants }\end{array}$ & $\begin{array}{l}\text { It does not comment on the communication and } \\
\text { application programming interfaces required in } \\
\text { the individual components of wind power plants. }\end{array}$ & $\begin{array}{l}\text { Provide the recommendations for enabling communi- } \\
\text { cation between wind power plants and the external } \\
\text { server. }\end{array}$ \\
\hline IEC 62052-11:2003 & $\begin{array}{l}\text { Electricity metering equipment } \\
\text { (AC) - General requirements, tests, } \\
\text { and test conditions }\end{array}$ & $\begin{array}{l}\text { Meter communication paradigm with the service } \\
\text { provider or to the installed infrastructure is not } \\
\text { discussed. }\end{array}$ & $\begin{array}{l}\text { Provide specifications for data transfers to the ser- } \\
\text { vice provider by considering the infrastructure-specific } \\
\text { communication paradigm. }\end{array}$ \\
\hline IEC 62053 & $\begin{array}{l}\text { Electricity metering equipment } \\
(\mathrm{AC})\end{array}$ & $\begin{array}{l}\text { Essence for update in order to provide similar } \\
\text { guidelines for smart meters. }\end{array}$ & $\begin{array}{l}\text { It should list the interface requirements for transmit- } \\
\text { ting the gathered values securely from meters to the } \\
\text { utility provider. }\end{array}$ \\
\hline CLC/TS 50549 & $\begin{array}{l}\text { Generating power plants to con- } \\
\text { nect in parallel with distribution net- } \\
\text { works }\end{array}$ & $\begin{array}{l}\text { No course of action for DRESs integration at the } \\
\text { generator level }\end{array}$ & $\begin{array}{l}\text { 1) It should outline the details for DRESs penetration. } \\
\text { 2) It should specify the details on how to calculate its } \\
\text { impact on the overall assessment of the power system. }\end{array}$ \\
\hline EVS 50438:2013 & $\begin{array}{l}\text { Micro-generating plants to connect } \\
\text { in parallel with pubic low-voltage } \\
\text { distribution networks }\end{array}$ & $\begin{array}{l}\text { No consideration of how communication signals } \\
\text { will traverse from the micro-generating plants to } \\
\text { the grid server. }\end{array}$ & $\begin{array}{l}\text { Required to address the issue related to the communi- } \\
\text { cating signals while integrating DRESs in the micro- } \\
\text { grid level. }\end{array}$ \\
\hline
\end{tabular}

While often not being the directly stated purpose, the standards and regulations discussed in this paper provide a good basis for DRESs integration in the smart grid to support various services in smart cities. However, the successful integration of DRESs is feasible only if the standards consider the end-to-end data transmission security, grid resilience, and assurance of the grid's real-time optimal performance and stability. In parallel, the standards need to consider the aspect of interoperability for various devices, operators, and services to exchange information in a seamless manner.

\section{CONCLUSION}

This paper outlines the relevant international standards available in the electricity market for the purpose of integrating DRESs, while ensuring the smooth working of the future electricity distribution network. These standards provide detailed information on the integration of various energy sources in different components of the grid, with some of these standards also describing how the signals are processed at the local controller. However, most of the present standards lack the specifications or recommendations on the integration of DRESs at the different levels of the distribution system in a unified manner and processing of their communication signals to the control server in real-time. We recommend that the relevant standards be further studied with a view to revising or even augmenting them to meet the needs of the future electricity distribution network.

\section{Acknowledgement}

The authors acknowledge the support of the H2020 EU's Enable Ancillary Services by Renewable Energy Sources
(EASY-RES) project (Agreement No.764090) for funding this research work. More information about the project can be found on http://www.easyres-project.eu/.

\section{REFERENCES}

[1] V. C. Gungor, D. Sahin, T. Kocak, S. Ergut, C. Buccella, C. Cecati, and G. P. Hancke, "Smart grid technologies: Communication technologies and standards," IEEE Transactions on Industrial Informatics, vol. 7, no. 4, pp. 529-539, Nov 2011.

[2] "IEEE standard for interconnection and interoperability of distributed energy resources with associated electric power systems interfaces," IEEE Std 1547-2018, Febuary 2018, Available: https://standards.ieee.org/standard/1547-2018.html.

[3] "IEEE standard definitions for the measurement of electric power quantities under sinusoidal, nonsinusoidal, balanced, or unbalanced conditions," IEEE Std 1459-2010, pp. 1-50, March 2010.

[4] "IEEE guide for voltage sag indices," IEEE Std 1564-2014, March 2014, Available: https://standards.iee.org/standard/1564-2014.html.

[5] "Wind turbines - part 25-2: Communications for monitoring and control of wind power plants - information models," IEC 61400-25-2:2015, June 2015, Available: https://webstore.iec.ch/publication/22813.

[6] "Electricity metering equipment (ac) - general requirements, tests and test conditions - part 11: Metering equipment," IEC 62052-11:2003, Febuary 2015, Available: https://webstore.iec.ch/publication/6379.

[7] "Establishing a network code on requirements for grid connection of generators," Commission Regulation (EU) 2016/631, April 2016, Available: https://eur-lex.europa.eu/legalcontent/EN/TXT/?uri=CELEX\%3A32016R0631.

[8] "Requirements for micro-generating plants to be connected in parallel with public low-voltage distribution networks," EVS-EN 50438:2013, April 2016, Available: https://www.evs.ee/products/evs-en-50438-2013. 